\title{
Research on Refractive Index and Polarization Direction of light in Crystal
}

\author{
Yuqing Xie ${ }^{1, a}$, Chuanyang Wang ${ }^{1,2, b}$ and Dong Xue ${ }^{1,3, c^{*}}$
}

\author{
${ }^{1}$ School of Opto-Electronic Engineering, Zaozhuang University, Zaozhuang277160, China \\ ${ }^{2}$ School of Mechanical and Electrical Engineering, Zaozhuang University, Zaozhuang277160, \\ China \\ ${ }^{3}$ Zaozhuang Engineering Research Center of Terahertz, Zaozhuang 277160, China \\ a harrison.xie@outlook.com, b904933380@qq.com, ' fiberlaser@126.com \\ * the corresponding author
}

Keywords: Refractive index; Vibration; Polarization; Uniaxial crystal

\begin{abstract}
The optical indicatrix gives the relation between the direction of vibration and the index of refraction of light propagating in the crystal. In this paper, from the direction of the wave normal and the optical indicatrix, the general formula of the refractive index and the direction of the vibration of the light propagating in the crystal is derived by using the coordinate rotation method. The solution is appropriate to both uniaxial crystal and biaxial crystal. The result is simple and meaningful with physical significance.
\end{abstract}

\section{Introduction}

The optical indicatrix is a description of the crystallographic optical characteristics of an important mathematical model in crystallographic Optics. It gives the relation between the direction of wave normal, the direction of vibration and the index of refraction when the light propagates in the crystal. Many important researches on crystal optics and optical properties should begin with the optical indicatrix. In this paper, from the direction of the wave normal and the optical indicatrix, the general formula of the refractive index and the direction of the vibration of the light propagating in the crystal is derived by using the rotation of the coordinate axis. The solution to the existing data is only about one axis crystal, avoiding the two axis crystal, or derived from the first equation of crystallographic optics. The process of solution is deep complex, and not easy to grasp. In this paper, the relation between the direction of the wave normal and the direction of vibration and the refractive index is given visually by the rotation of the coordinate axis.

\section{Theoretical basis}

The optical indicatrix is a rotating ellipsoid whose refractive index is expressed in the direction of light wave vibration by a line segment of a certain length, or the three axis ellipsoid. And it is a single shell surface. Assuming that the direction of the wave normal is known, the two refractive index values in this direction can be solved by making a plane perpendicular to the known wave normal through the center of the optical indicatrix. The plane cut out from the optical indicatrix is absolutely a elliptic curve (It is a circle curve in particular). The long and the short diameter of the ellipse represent the magnitude of the two refractive index. The direction of the long and short radius of the ellipse represents the direction of vibration of the two light wave. The two directions are perpendicular to each other and are perpendicular to the direction of the wave normal.

The optical indicatrix of an advanced crystal family is a sphere, and there is no birefringence occurs when light passes through. The equations are: $x^{2}+y^{2}+z^{2}=n^{2}$.

The optical indicatrix of an intermediate crystal (uniaxial) is a rotational ellipsoid whose equation is: $\mathrm{x}^{2} / \mathrm{n}_{\mathrm{o}}{ }^{2}+\mathrm{y}^{2} / \mathrm{n}_{\mathrm{o}}^{2}+\mathrm{z}^{2} / \mathrm{n}_{\mathrm{e}}^{2}=1$.

The light body of the lower crystalline family (two axis) is the three axis ellipsoid, and the equation is : $\mathrm{x}^{2} / \mathrm{n}_{\mathrm{g}}^{2}+\mathrm{y}^{2} / \mathrm{n}_{\mathrm{m}}{ }^{2}+\mathrm{z}^{2} / \mathrm{n}_{\mathrm{p}}{ }^{2}=1$. 


\section{Formula Deduction}

In order to preserve the generality, we formulate the equation of the ellipsoid of optical indicatrix as: $\mathrm{x}^{2} / \mathrm{a}^{2}+\mathrm{y}^{2} / \mathrm{b}^{2}+\mathrm{z}^{2} / \mathrm{c}^{2}=1$. The normal direction of the incident light wave does not coincide with the axis, and the directional derivative is $\mathrm{s}$ : $\quad(\mathrm{A}, \mathrm{B},-1)$. Thus, the plane perpendicular to the normal direction s of the light wave at the origin of the coordinate is $\pi: \mathrm{z}=\mathrm{Ax}+\mathrm{By}$. An ellipse is obtained by intersecting the plane with the ellipsoid. The following equations are described as:

$$
\left\{\begin{array}{l}
\frac{x^{2}}{a^{2}}+\frac{y^{2}}{b^{2}}+\frac{z^{2}}{c^{2}}=1 \\
z=A x+B y
\end{array} .\right.
$$

Here we turn the ellipse into a canonical form by rotation of the coordinate axis. We rotate the original $\mathrm{x}$ axis to the $\mathrm{x}^{\prime}$ axis under the new coordinate, which is consistent with $\mathrm{s}$. And the direction of the $\mathrm{x}^{\prime}, \mathrm{y}^{\prime}$ axis is arbitrary. Now, the ellipse is in the plane $\mathrm{y}^{\prime} \mathrm{oz}$.

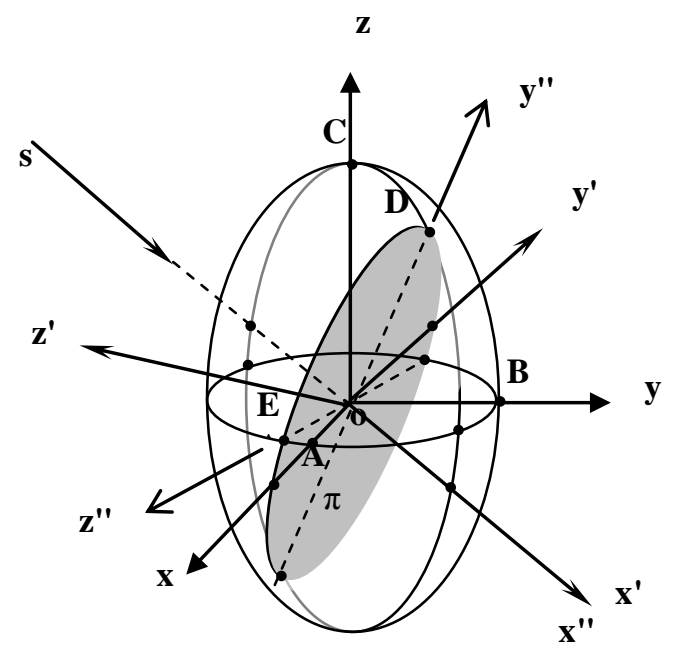

Figure. 1. The rotation of three-dimension coordinate

As shown in Fig. 1, the original coordinate system is a xoy system; after one rotations, the coordinate system is $\mathrm{x}$ 'oy'; after 2 rotations the coordinate system is $\mathrm{x}$ "oy". $\mathrm{OA}=\mathrm{a}, \mathrm{OB}=\mathrm{b}$ and $\mathrm{OC}=\mathrm{c}$ are the three main radii of the ellipsoid (corresponding to the 3 principal refractive index of polarized light); OD and $\mathrm{OE}$ are the long and minor axes of the ellipse (corresponding to the 2 refractive indices of the $s$ ). According to linear transformation:

$$
\left\{\begin{array}{l}
x^{\prime}=\frac{1}{l}(A x+B y-z) \\
y^{\prime}=\frac{1}{m}(y+B z) \\
z^{\prime}=\frac{1}{l m}\left[\left(1+B^{2}\right) x-A B y+A z\right]
\end{array},\right.
$$

where

$$
\left\{\begin{array}{l}
l=\sqrt{1+A^{2}+B^{2}} \\
m=\sqrt{1+B^{2}}
\end{array} .\right.
$$

And the inverse transform is: 


$$
\left\{\begin{array}{l}
x=\frac{1}{l}\left(A x^{\prime}+m z^{\prime}\right) \\
y=\frac{1}{l m}\left(B m x^{\prime}+l y^{\prime}-A B z^{\prime}\right) . \\
z=\frac{1}{l m}\left(-m x^{\prime}+B l y^{\prime}+A z^{\prime}\right)
\end{array}\right.
$$

Put (3) into (1) formula, and get the elliptic equation:

$$
\left\{\begin{array}{l}
x^{\prime 2}\left(D A^{2}+E B^{2} m^{2}+F A B m\right)+y^{\prime 2} E l^{2}+z^{\prime 2}\left(D m^{2}+E A^{2} B^{2}-F A B m\right) \\
+x^{\prime} y^{\prime}(2 E B m l+F A l)+x^{\prime} z^{\prime}\left(2 A D m-2 A B^{2} E m-F A^{2} B+F B m^{2}\right) \\
+y^{\prime} z^{\prime}(F l m-2 E A B l)-1=0 \\
x^{\prime}=0
\end{array}\right.
$$

where

$$
\mathrm{D}=\frac{1}{l^{2}}\left(\frac{1}{a^{2}}+\frac{A^{2}}{c^{2}}\right) ; \mathrm{E}=\frac{1}{l^{2} m^{2}}\left(\frac{1}{b^{2}}+\frac{B^{2}}{c^{2}}\right) ; \mathrm{F}=\frac{2 A B}{l^{2} m^{2} c^{2}} .
$$

The elliptic equation at this point can be expressed as:

$$
\mathrm{Gy}^{\prime 2}+\mathrm{Hz}^{\prime 2}+\mathrm{Iy}^{\prime} \mathrm{z}^{\prime}-1=0
$$

where

$$
\mathrm{G}=\mathrm{E} l^{2} ; \mathrm{H}=\mathrm{Dm}^{2}+\mathrm{EA}^{2} \mathrm{~B}^{2}-\mathrm{FABm} ; \mathrm{I}=\mathrm{F} l \mathrm{~m}-2 \mathrm{EAB} l .
$$

The plane $x^{\prime} y^{\prime} z^{\prime}$ rotates $\theta$ degrees around the $x^{\prime}$ axis (Fig. 2). The transformation is

$$
y^{\prime}=y^{\prime \prime} \cos \theta-z " \sin \theta, \quad z^{\prime}=y^{\prime \prime} \sin \theta+z^{\prime \prime} \cos \theta \text {. }
$$

By (6), when the rotation angle $\theta$ satisfies the following expression:

$$
\operatorname{tg} 2 \theta=\mathrm{I} /(\mathrm{G}-\mathrm{H}) \text {, }
$$

the original type of the two cross terms can be removed, (6) change to:

$$
y^{\prime \prime}(G \cos 2 \theta+H \sin 2 \theta+I \sin \theta \cos \theta)+z^{\prime \prime}(G \sin 2 \theta+H \cos 2 \theta+I \sin \theta \cos \theta)=1 \text {. }
$$

This is the standard equation of the ellipse we require. And its long diameter and short diameter are respectively $a^{\prime}$ and $b^{\prime}$, there are:

$$
1 / \mathrm{a}^{2}=\mathrm{G} \cos ^{2} \theta+\mathrm{H} \sin ^{2} \theta+\mathrm{I} \sin \theta \cos \theta, \quad 1 / \mathrm{b}^{\prime 2}=\mathrm{G} \sin ^{2} \theta+H \cos ^{2} \theta+\mathrm{I} \sin \theta \cos \theta .
$$

The $y^{\prime \prime}$ axis and the $z^{\prime \prime}$ axis are the spatial directions of the long diameter a' and short diameter $b^{\prime}$.

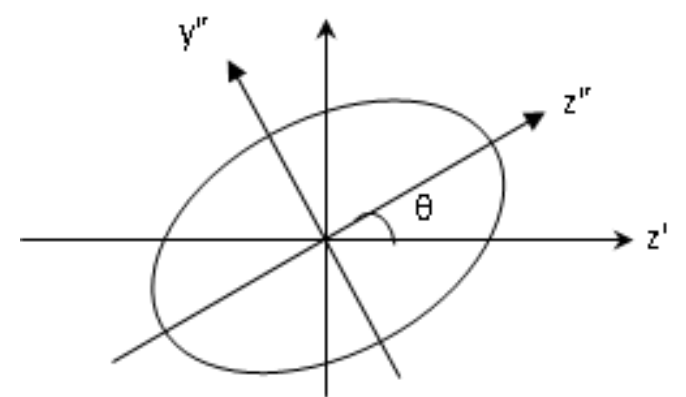

Figure. 2. The rotation of two-dimension coordinate

\section{Conclusion}

The method mentioned above is applicable to various situations of the refractive index of light waves propagating in crystals and the direction of vibration.

The $\mathrm{x}$ " axis is the direction of the known light wave normal; the axis $\mathrm{y}^{\prime \prime}$ and axis $\mathrm{z}$ " represent the vibration direction of the two polarized lights after birefringence; while the long and short diameter indicate the refractive index of the polarized light in that direction. When the normal direction of the light wave travels in the direction of the main axis ( $\mathrm{x}$ axis) of the optical indicatrix, it can be intuitively obtained that the elliptic equation corresponding to it is $\mathrm{y}^{2} / \mathrm{b}^{2}+\mathrm{z}^{2} / \mathrm{c}^{2}=1$. At this time, the vibration direction of a bundle of polarized light is $y$ axis, the refractive index is $b$; the vibration direction of 
another beam of polarized light is $\mathrm{z}$ axis, and the refractive index is $\mathrm{c}$. The same is true when the normal direction of light waves travels along the $\mathrm{y}$ axis or the $\mathrm{z}$ axis.

The above formula is derived from lower crystalline family (two axis crystal). There are three main refractive indexes: $a, b$ and $c$. Making two of them equal, such as $a=b \neq c$, we can solve the intermediate crystal family (uniaxial) equation. And when $\mathrm{a}=\mathrm{b}=\mathrm{c}$, the ellipsoid degenerates into a sphere, and the intersection of the plane $\pi$ is a circle, There is no difference between the long and short diameter. The polarization direction of the light is arbitrary, and the refractive index is constant. That is, no birefringence occurs, which is the basic property of the higher crystalline family.

In the lower crystalline family (two axis), we might as well take a special direction for checking; That is, the light wave normal takes place in the direction of the first optical axis $\mathbf{s}: \quad\left(\frac{c}{a} \sqrt{\frac{a^{2}-b^{2}}{b^{2}-c^{2}}}, 0, \pm 1\right)$ $($ set $\mathrm{a}>\mathrm{b}>\mathrm{c})$. The plane perpendicular to the normal direction $\mathrm{s}$ of the light wave is $\pi: \mathrm{z}=\frac{c}{a} \sqrt{\frac{a^{2}-b^{2}}{b^{2}-c^{2}}} \mathrm{x}$. That is, put $\mathrm{A}=\frac{c}{a} \sqrt{\frac{a^{2}-b^{2}}{b^{2}-c^{2}}}, \mathrm{~B}=0$ in (1) formula into (2). We can obtain $l=\frac{b}{a} \sqrt{\frac{a^{2}-c^{2}}{b^{2}-c^{2}}} ; m=1$.Substituting (5) is $D=\frac{1}{b^{2}} ; E=\frac{a^{2}\left(b^{2}-c^{2}\right)}{b^{2}\left(a^{4}-c^{4}\right)} ; F=0$, Substituting (7) is: $G=\frac{1}{b^{2}} ; \quad H=\frac{1}{b^{2}} ; I=0$. At this point, the ellipse has been reduced to a circle without rotating the fixed axis.

Its physical significance is obvious: when the light wave normal goes along this direction, no birefringence occurs, and the two refractive index is $b$. That is, the main refractive index (nm) of the lower crystalline family (two axis). This is the basic property of light propagating along the first axis.

\section{Acknowledgement}

This work is supported by national natural science fund project (No. 11447200); Shandong provincial natural science foundation (No.ZR201702200400); Science and technology program of Shandong higher education institutions (No. J17KA087); The program of independent innovation and achievement transformation plan for Zaozhuang (No. 2016GH19); Science and technology program of Zaozhuang (No. 2016GX31).

\section{References}

[1] S.N. Savenkov, Jones and Mueller matrices: structure, symmetry relations and information content.( 2009) 71-119.

[2] L. Jinjun, L. Xiangyang, Y. Qiao, S. Xueping, G. Xiaochuan, Comparison and analysis on Polarization aberration in Pehan prisms and the Telescope system including Pehan prisms, Physics Procedia.19 (2011) 436-441.

[3] T.J. Moore, M.R. Jones, D.R. Tree, D.D. Allred, An inexpensive high-temperature optical fiber thermometer, Journal of Quantitative Spectroscopy and Radiative Transfer.187 (2017) 358-363, 2017.

[4] A. Yariv, P. Yeh, Optical waves in crystals, WILEY, New York, 1984.

[5] D. Goldstein, Polarized Light, Third Edition, Crc Press, New York, 2010.

[6] S.N. Savenkov, Jones and Mueller matrices: structure, symmetry relations and information content, Light Scattering Reviews.4 (2009) 71-119.

[7] S.S. Ivanov, A.A. Rangelov, N.V. Vitanov, T. Peters, T. Halfmann, Highly efficient broadband conversion of light polarization by composite retarders, Journal of the Optical Society of America :A Optics Image Science \& Vision. 29 (2012) 265-269. 
[8] R.C. Jones, A New Calculus for the Treatment of Optical SystemsI. Description and Discussion of the Calculus, J. Opt. Soc. Am.31 (1941) 488-493. 